\title{
Ocorrência de dentes supranumerários em pacientes do serviço de Cirurgia e Traumatologia Buco-Maxilo-Facial, Faculdade de Odon- tologia da UFRGS, no período de 1998 a 2001 Occurrence of supranumerary teeth from the patients of the Department of Surgery, Faculty of Dentistry of the UFRGS, from 1998 to 2001
}

\author{
João Julio da Cunha Filho* \\ Edela Puricelli** \\ Tatiana Wahl Hennigen*** \\ Mauro Gomes Trein Leite*** \\ Maurício Assunção Pereira*** \\ Gustavo Lisboa Martins****
}

\begin{abstract}
RESUMO
Este trabalho apresenta uma revisão de 848 radiografias panorâmicas dos arquivos da Disciplina de Cirurgia e Traumatologia BucoMaxilo-Facial, da Faculdade de Odontologia da Universidade Federal do Rio Grande do Sul, com a finalidade de avaliar a presença de dentes supranumerários, e suas características quanto ao sexo, localização, forma, situação no arco dentário, número de rázes e idade. 0 propósito deste trabalho foi comparar os dados encontrados com a literatura e analisar a prevalência de dentes supranumerários nessa população, segundo as características mencionadas. Foram encontrados 64 dentes supranumerários, em 54 pacientes, sendo a região anterior da maxila a de maior ocorrência, apresentando $37(57,8 \%)$ do total dos casos de dentes supranumerários, com prevalência de dentes conóides e retidos, sem patologias locais associadas.Os resultados encontrados na população estudada estão de acordo com os achados científicos publicados na literatura.
\end{abstract}

PALAVRAS-CHAVE

Supranumerário; Radiografia panorâmica; Dente retido

\section{INTRODUÇÃO}

A presença de dentes supranumerários na população em geral tem apresentado uma prevalência importante, capaz de despertar o desenvolvimento de meios diagnósticos, que a todo o momento evoluem e permitem variações técnicas com maior precisão na localização destes dentes.

O grande número de casos, diagnosticados e tratados pela Disciplina de Cirurgia e Traumatologia Buco-Maxilo-Facial da Faculdade de Odontologia da UFRGS, fez com que estudássemos estes pacientes, quanto à prevalência de algumas de suas características.

Dente supranumerário ou hiperdontia é definido como o excesso do número de dentes quando comparado à dentição normal da população. As classificações são baseadas no tipo de dentição, quanto à posição no arco dentário, a forma, número de raízes e se eruptados ou retidos. Vários conflitos de resultados são apresentados por estudos epidemiológicos, sendo atribuído tais diferenças a técnicas e critérios diagnósticos e, principalmente, aos diferentes grupos raciais.
Assim, a proposição deste trabalho é:

- avaliar epidemiologicamente a incidência de dentes supranumerários no período de 1998 a 2001, examinando 848 radiografias panorâmicas do arquivo da Disciplina de CTBMF, desta Faculdade.

- avaliar a prevalência das principais características dos dentes supranumerários, quanto à forma, número, retenção, sexo, idade média, espessamento de folículo pericoronário e localização anatômica de maior ocorrência.

\section{REVISÃO DA LITERATURA}

Segundo ZHU et al. (1996), o primeiro relato de dente supranumerário apareceu entre 23 e 79 a.C.. São considerados como um aumento no número de dentes presentes na dentição, excedendo o número normal da população (TIMOCIN et al., 1994).

Os dentes supranumerários ocorrem na população em geral, segundo HOU et al. (1995), entre $0,31 \%$ e $6,6 \%$, com alguma variação destes índices em outros trabalhos. Em crianças esta incidência é de $0,2 \%$, segundo JONES et al. (1993) e em adultos jovens, de acordo com o estudo realizado por
PELTOLA et al. (1997), está em torno de $3 \%$. A ocorrência de dentes supranumerários acomete caucasianos entre 1,0 e 3,1\% (KING et al., 1993; TROTMAN et al., 1994). Segundo HAAVIKKO (1971), a população asiática é acometida com alta incidência de supranumerários. Os portadores da Síndrome de Down apresentam uma incidência entre 2,3 e $3,4 \%$ (KING et al., 1993) de dentes supranumerários. Há uma prevalência maior nos homens em relação às mulheres (TIMOCIN et al., 1994; KAYALIBAY et al., 1996; NOIKURA et al., 1996), podendo chegar até a proporção de 6,5:1 (KING et al., 1993).

0 aparecimento de dentes supranumerários, de acordo com ZHU et al. (1996), pode se classificar como prédecíduo, similar permanente ou suplementar e pós- permanente, quando este for de desenvolvimento tardio ao permanente. KING et al. (1993) sugerem que o desenvolvimento tardio de dentes supranumerários esteja relacionado com uma terceira dentição ou pós-permanente.

Podem ocorrer individualmente (MELNIK 1993; SCHATZ et al., 1994; ZHU et al.,

* Professor Assistente do Departamento de Cirurgia e Ortopedia, FO-UFRGS; Mestre em CTBMF, FO-PUCRS. ** Doutora pela Universidade de Düsseldorf, Alemanha; Profa. Curso de Mestrado em Clínicas Odontológicas -Área de Concentração em CTBMF, FO-UFRGS; Chefe da Unidade de CTBMF-Hospital de Clínicas de PA/HCPA-UFRGS. *** Cirurgião Dentista; Aluno do Programa de Capacitação em CTBMF da FO-UFRGS. $* * * *$ Mestre em CTBMF, FO-UFRGS. 
1996) ou de forma múltipla (CHADWICK et al.1993; KING et al., 1993; TASAR et al., 1995; ATASU et al., 1996; ZHU et al., 1998), unilaterais ou bilaterais (TIMOCIN et al., 1994), sendo na maioria únicos (KING et al., 1993). ZHU et al. (1996) relatam que supranumerários únicos ocorrem entre $76 \mathrm{e}$ $86 \%$, aos pares entre 12 e $23 \%$ e três ou mais (múltiplos) em 1\%.

Os dentes supranumerários podem estar não erupcionados em 80\% (TIMOCIN et al., 1994). ZHU et al. (1996), em seu trabalho, sugerem que $75 \%$ dos dentes supranumerários na região anterior encontram-se não erupcionados e $25 \%$ destes estão parcial ou totalmente erupcionados.

Sua ocorrência na dentição permanente é mais comum (KING et al., 1993; NOIKURA et al., 1996) e encontrados, na população geral, entre 1 e 5\% (CHADWICK et al., 1993). Na dentição decídua a sua ocorrência é rara, variando de 0,1 a $1 \%$ de acordo com estudo realizado por NIK-HUSSEIN et al. (1996). A ocorrência de dentes supranumerários em ambas dentições em um mesmo indivíduo apresenta uma ocorrência em torno de 30\% (ZHU et al., 1996).

Em relação às arcadas dentárias, os dentes supranumerários podem ocorrer na maxila, na mandíbula ou em ambas arcadas (TIMOCIN et al., 1994). De acordo com TROTMAN et al. (1994) e ZHU et al. (1996), dentes supranumerários ocorrem com maior frequiência na maxila, totalizando entre 90 e $98 \%$ do total de dentes supranumerários.

Os dentes supranumerários podem ser encontrados em qualquer área dos arcos dentais (KING et al., 1993). A região mais comum é a anterior maxilar (CHADWICK et al., 1993; TROTMAN et al., 1994; TIMOCIN et al., 1994), seguida das regiões de prémolares inferiores, molares superiores e caninos (KING et al., 1993).

Alguns trabalhos relatam ainda a ocorrência de dentes supranumerários nas cavidades nasais, órbita, seio maxilar, ovários (TIMOCIN et al., 1994), palato mole, sutura incisiva, concha oftálmica, sutura esfenomaxilar, tuberosidade maxilar e até entre a órbita e o cérebro (ZHU et al., 1996)

Ocorrem em diversos tipos, classificados de mesiodens, paramolares, distomolares (CHADWICK et al., 1993) e impactados (HOU et al., 1995; ZHU et al., 1996). Os mesiodens, localizados na prémaxila entre os incisivos centrais superiores (CASTILHO et al., 1997), são os mais comuns (TIMOCIN et al., 1994; KAYALIBAY et al.,1996; NOIKURA et al., 1996), totalizando entre 45 e $67 \%$ de todos os dentes supranumerários (ZHU et al., 1996). O quarto molar ou distomolar totaliza $28,2 \%$ (TIMOCIN et al., 1994), os prémolares 8 a 10\% (HEDGE et al., 1996;
ZHU et al., 1996) e os molares inferiores $2 \%$, seguidos de caninos e incisivos laterais (TIMOCIN et al., 1994). Podem ocorrer ainda paramolares, totalizando $11,6 \%$ do número de supranumerários (TIMOCIN et al., 1994). Quando os dentes supranumerários ocorrem em ambas arcadas, a região de pré-molares é atingida com maior freqüência, com $62,1 \%$ dos dentes, seguido da região de molares com $27,6 \%$ e da região anterior, com $10,3 \%$ do total de dentes (TIMOCIN et al., 1994).

Quanto à forma, podem ser encontrados com aspecto de agulha, tuberculado, suplementar e odontoma (CHADWICK et al., 1993), podendo ainda ter o aspecto rudimentar, segundo KING et al. (1993) e por ZHU et al. (1996). CASTILHO et al. (1997), sugere a classificação quanto à forma em cônicos (pequenos, coniformes e com raiz em desenvolvimento normal); tuberculares (multicuspídeos, pequenos, com raiz rudimentar); suplementares (iguais aos dentes da série); e odontomas (sem formato regular).

$\mathrm{O}$ diagnóstico de dentes supranumerários se dá de forma mais satisfatória unindo exame clínico com exame radiográfico (HOU et al., 1995), porém a maioria pode ser encontrado somente por meio de radiografias (HEDGE et al., 1996). Achados ocasionais ou ausência de dentes permanentes no arco dentário, no período cronologicamente devido, podem estar associados a dentes supranumerários. Segundo PEREIRA, PURICELLI (1981) e PURICELLI, CLOSS, BERTHOLD, (1991), a ausência de um dente no arco dentário pode sugerir a presença de um dente supranumerário, mas não se deve descartar a possibilidade do traumatismo na dentição decídua, acarretando a dilaceração corono-radicular.

Vários autores determinam as possíveis causas do aparecimento de dentes supranumerários. A teoria da hiperatividade da lâmina dental como formadora de novos germes dentários é a mais aceita (KING et al., 1993; TROTMAN et al., 1994; TIMOCIN et al. 1994; TASAR et al., 1995; KAYALIBAY et al. 1996; ZHU et al., 1996). $\mathrm{O}$ atavismo, teoria que levanta a possibilidade de ancestrais humanos terem maior número de dentes, também é relatada por alguns autores (KING et al., 1993; TIMOCIN et al., 1994; ZHU et al., 1996).

Outra hipótese seria a dicotomia do germe dentário, levantada por KING et al. (1993) e por ZHU et al. (1996). Estes autores também sugerem que a combinação entre fatores genéticos e fatores ambientais podem gerar dentes supranumerários. NOIKURA et al. (1996) sugerem fator morfogenético no processo de odontogênese.

TIMOCIN et al. (1994) levantam a possi- bilidade de herança ligada ao sexo. KRONMILLER (1995) defende ação de um Fator de Crescimento Epidermal (EGF) como necessário para a iniciação da odontogênese e afirma que o EGF exógeno altera o padrão da lâmina dental, podendo provocar odontogênese de supranumerários. Porém, a real etiologia ainda é desconhecida (TROTMAN et al., 1994).

A relação entre dentes supranumerários e síndromes é descrita por vários autores. A síndrome de Gardner (CHADWICK et al., 1993; KING et al., 1993; TROTMAN et al., 1994; TIMOCIN et al., 1994; ZHU et al., 1996), a Displasia ou Disostose Cleidocranial (CHADWICK et al., 1993; KING et al. 1993; TROTMAN et al., 1994; TIMOCIN et al., 1994; TASAR et al., 1995; HEDGE et al., 1996; ATASU et al.,1996; ZHU et al., 1996; BECKER et al., 1997), o Palato e/ou Lábio Fendido (CHADWICK et al., 1993; ZHU et al.1996 ; VICHI et al., 1996), a síndrome de Nance-Horan (TROTMAN et al., 1994), a síndrome de Treacher Collins (ANIL et al., 1995) são algumas das síndromes citadas na bibliografia.

CHOW (1997) relata um de caso com síndrome de Down, onde a hipodontia e hiperdontia é uma de suas manifestações clínicas, sendo a presença concomitante destes sinais relativamente rara. 0 manejo ortodôntico precoce, associado a um acentuado controle de higiene oral é uma forma de prevenção para problemas futuros, já que a erupção dentária se apresenta de forma tardia. A presença de raízes curtas, um reduzido número de dentes e o espaço do arco dentário são problemas aos quais os profissionais devem estar atentos.

ATASU et al. (1996) descreveram algumas características clínicas encontradas em um paciente portador de disostose cleidocraniana com múltiplos dentes supranumerários. Os autores relatam a disostose como uma alteração autossômica dominante de alta penetração atingindo a formação esqueletal e o desenvolvimento dentário. Os indivíduos apresentam hipoplasia clavicular, baixa estatura e suturas ósseas não consolidadas. A presença de dentes supranumerários é freqüente. $\mathrm{O}$ autor relata um caso de hiperdontia na região anterior da maxila anterior e na região de pré-molares, com forma rudimentar e do tipo conóide, associado a deslocamento e retenção dentária.

A tabela a seguir exemplifica mais detalhadamente a relação das síndromes que apresentam, entre suas características, a presença de dente (s) supranumerário (s), com as respectivas possibilidades de ocorrência destes:

Os dentes supranumerários podem ter 1 EGF- Epidermal Growth Factor 


\section{SÍNDROME}

\section{FREQÜÊNCIA RELATIVA}

Síndrome de Apert

Cromossomo 13

Displasia Cleidocraneal

Displasia Craniometafisica

Doença de Crouzon

Síndrome de Down

Síndrome de Ehlers-Danlos

Fucosidose

Síndrome de Gardner

Síndrome de Hellerman-Streiff

Efeitos dentais da Hipertricose

Síndrome de Klippel-Trenaunary-Weber

Síndrome Laband

Retardamento mental, macrocefalia, hipotonia, coarse fácies

Retardamento mental, pterigia, distinctive fácies

Síndrome de Nance-Horan

Síndrome Oro-Facial-Digital (tipos 1 e 3)

Polidactilia

Síndrome Sturge-Weber

Síndrome de Sobre-crescimento tipo Teebi

Síndrome Tricô-rino-falangeal

Retardamento mental ligado ao cromossomo X, Crescimento Marfanóide

Retardamento mental ligado ao cromossomo X, Tipo Prieto

Fonte: Zhu et al., 1996

vários destinos. Podem permanecer retidos, não gerando efeitos na dentição permanente (TROTMAN et al., 1994; TIMOCIN et al., 1994, YAMAOKA et al., 1995), o que ocorre, segundo ZHU et al. (1996), entre 7 a $20 \%$ dos casos. Podem interferir nos dentes permanentes, ocasionando atraso na erupção (HOU et al., 1995) em cerca de 28 a $60 \%$ dos casos, impactação (YAMAOKA et al., 1995-a), maloclusão (TROTMAN et al., 1994; CANUT 1996), apinhamento, diastema (HOU et al., 1995; YAMAOKA et al., 1995a), rotação (HOU et al., 1995; ZHU et al., 1996), reabsorções radiculares (HOU et al., 1995; YAMAOKA et al., 1995-a; ZHU et al., 1996), ou até mesmo erupcionar, como já citado anteriormente.

Alguns autores citam patologias relacionadas aos dentes supranumerários. ZHU et al. (1996) apontam o aparecimento de cistos dentígeros, também citado por $\mathrm{HOU}$ et al. (1995) ou primordiais, enquanto que TIMOCIN et al. (1994) sugere também o aparecimento de neoplasias. Periodontite (HOU et al., 1995) e cárie no dente adjacente (ZHU et al., 1996), assim como reabsorções da dentição decídua e permanente (GELLIN, 1984), também foram relatadas.

Outras patologias também podem estar associadas a dentes supranumerários. A fusão dentária foi observada por ZMENER (1995), KAYALIBAY et al. e ZHU et al. (1996), descrita por ATASU et al. (1996) como sinodontia. Dens in dente e dens invaginatus foram descritas no trabalho de NOIKURA et al. (1996).

O tratamento dos dentes supranumerários foi descrito por diversos autores, onde foram encontrados trabalhos sugerindo tratamento cirúrgico-ortodôntico, tratamento ortodôntico e tratamento cirúrgico único e exclusivo, além de acompanhamento clínico-radiográfico dos dentes supranumerários.

$\mathrm{O}$ tratamento cirúrgico de exodontia dos dentes supranumerários é o de escolha (KING et al. 1993; ZHU et al. 1996; RIZZUTI et al., 1997). Deve acontecer o mais cedo possível (YAMAOKA et al., 1995-b), com idade preferencial entre 8 e 10 anos. 0 autotransplante pode ser uma outra alternativa de tratamento cirúrgico, citado por SCHATZ et al. (1994). O tratamento cirúrgico pode gerar algumas conseqüências, como o rompimento do feixe vásculo-nervoso do dente adjacente e parestesias (KING et al., 1993).

MELNIK (1993) cita tratamento exclusivamente ortodôntico de um incisivo supranumerário, colocado no lugar de um dente fusionado. KING et al. (1993) e ZHU et al. (1996), sugerem o acompanhamento clínico-radiográfico dos dentes supranumerários, sem qualquer tratamento cirúrgico.

Os pacientes com história de supranumerários devem sofrer acompanhamento clínico e radiográfico regularmente (CHADWICK et al., 1993; KING et al., 1995). CHADWICK et al. (1993) sugerem radiografia de controle entre 16 e 18 anos de idade, que podem também ser utilizadas para verificar a situação dos terceiros molares, evitando complicações tardias pós-cirúrgicas (TIMOCIN et al., 1994).

Segundo BERGSTROM (1977) a ausên- cia congênita de dentes ocorre com maior prevalência em mulheres, na proporção de $3: 2$, envolvendo a dentição permanente, atingindo entre 3,5 a $8 \%$ da população, sem considerar os terceiros molares. A ausência de um ou mais terceiros molares acomete de 20,8 a $22,7 \%$ da população, segundo HAAVIKKO (1971) e LYNHAM (1989), ao analisarem radiografias panorâmicas.

Na população asiática a região de incisivos inferiores e nos caucasianos a região de pré-molares são comumente as que apresentam maior ausência de germes dentários, conforme os relatos de DIXON (1976), sendo constatada uma relação 13 vezes maior da ausência congênita de terceiros molares com outras ausências na dentição permanente. CRAIG (1964) relata mal formação coronária contra-lateral associada à ausência de um dos germes. BRIN et al.(1986) encontrou em seus estudos relação entre incisivos laterais conóides ou ausentes com a presença de caninos retidos. Relatos de VAN DER WEIDE et al. (1994) afirmam que em torno de 120 síndromes apresentam alterações no número de dentes.

A possibilidade de coexistirem as duas anomalias congênitas manifesta-se entre 8 a 10 casos para cada 10.000 pacientes, segundo MERCER (1970), enquanto GIBSON (1979) encontrou 20 casos em 4598 pacientes ortodônticos, com uma prevalência de 1:230 casos, sendo a etiologia da hiperdontia e da hipodontia desconhecida, apesar de algumas teorias serem levantadas, como a migração, proliferação e diferenciação de células neurais, e a interação entre células epiteliais e mesenquimais, durante o início da odontogênese.

RIZZUTI (1997) relata a ocorrência de 22 dentes permanentes supranumerários em posição ectópica em um caso clínico, sendo realizado o tratamento orto-cirúrgico durante sete anos. $\mathrm{O}$ autor relata que a interação multidisciplinar é fundamental para o sucesso do caso, principalmente devido ao longo período de acompanhamento. CHADWICK et al. (1993) relatam um caso onde quatro supranumerários foram removidos na região dos incisivos superiores, e após cinco anos pós-cirúrgicos identificaram-se outros quarto dentes supranumerários assintomáticos na região, através de controle radiográfico periódico, confirmando a necessidade de acompanhamento tardio dos pacientes que apresentam dentes supranumerários.

KING et al. (1993) relataram três casos clínicos com ocorrência de 20 dentes supranumerários na região de pré-molares, sendo 16 destes dentes com forma coronária e radicular compatível com os dentes da região, fazendo os autores afirmarem que tais dentes suplementares fazem parte de uma outra dentição, denominada pós-permanen- 
te. LOH (1993) relata um caso onde apresentam um dente supranumerário na região molar, com duas coroas e unirradicular. Duas teorias foram desenvolvidas: a primeira apresentada refere à possibilidade de um germe dentário com a divisão coronária. A segunda, apesar de ser considerada mais remota, considera a possibilidade de fusão de dois dentes supranumerários.

TIMOCIN et al. (1994) relataram casos de dentes supranumerários na região dos molares envolvendo os quatro quadrantes, em três pacientes. Em todos estes casos o tratamento cirúrgico foi realizado. Os autores alertam para o fato de dentes que apresentarem retenção ou atraso no período de erupção devem ser submetidos a uma investigação clínica-radiográfica, onde a hipótese de dente supranumerário seja cogitada.

JASMIM (1993) apresenta dois casos de pacientes gêmeos univitelinos, com idênticas retenções de dentes supranumerários na região de pré-molares inferiores, confirmando a característica genética monozigótica desta gestação.

\section{MATERIAL E MÉTODOS}

Para o presente estudo foram analisadas 848 radiografias panorâmicas do arquivo da Disciplina de Cirurgia e Traumatologia Bucomaxilo-Facial, no período de março de 1998 a agosto de 2001, de pacientes de ambos os sexos, e de todas as idades, com a finalidade de localizarmos através deste exame imagens compatíveis com dentes supranumerários. Somente casos de pacientes com radiografias panorâmicas foram incluídos neste estudo, não sendo considerado os pacientes portadores de síndromes com múltiplos dentes retidos, assim como os pacientes dos núcleos de implantodontia, prótese facial e ortodontia. A exclusão destes grupos devese ao fato de maior prevalência de mal formações genéticas sistêmicas e dentárias, fatores que estariam selecionando de maneira indesejada a amostra em estudo.

0 grupo de examinadores foi composto por três alunos e um professor do Programa de Capacitação em Cirurgia e Traumatologia Buco-Maxilo-Facial, da Faculdade de Odontologia da UFRGS.

Confeccionamos uma tabela de avaliação a ser preenchida pelos examinadores no decorrer da avaliação radiográfica. Cada exame radiográfico foi analisado individualmente sobre um negastoscópio de mesa, de medida de $20 \mathrm{~cm} \times 25 \mathrm{~cm}$, onde as radiografias eram colocadas e analisadas no sentido horário com a contagem dos dentes correspondente a faixa etária de cada paciente, com visão desarmada. Os casos de dúvida foram discutidos em grupo.

Pacientes com mais de uma radiografia panorâmica receberam análise individual em cada radiografia, sendo considerado apenas uma radiografia no estudo estatístico final, visto que se tratava do mesmo indivíduo.

A avaliação foi realizada seguindo a ordem alfabética do arquivo, com o registro das iniciais, pois possibilitou a recuperação do paciente naqueles casos onde os examinadores necessitaram uma maior análise. 0 registro das datas de nascimento permite a identificação criteriosa de cada paciente e o cálculo da idade do indivíduo. Após a colocação da radiografia sobre o negatoscópio, os examinadores deveriam realizar uma ampla varredura das regiões anatômicas envolvidas entre as articulações temporomandibulares e da sutura naso-frontal ao osso hióide. ram a possibilidade de dentes em localização ectópica. Após o exame referido cada
Desta forma, os examinadores elimina-

paciente foi submetido à contagem do número de dentes e verificada a presença de hipodontia ou hiperdontia. Caso fosse verificado um numero diferente do esperado cronologicamente, os examinadores deveriam passar a outro estágio, com maior detalhamento na inspeção, com o objetivo de conferir no exame panorâmico dente a dente, a existência ou não de dentes supranumerários. Os dados foram tabulados manualmente por cada examinador, ao final de cada exame. Ao final da tabulação as informações foram repassadas ao computador utilizando-se o programa Excel ${ }^{\otimes^{2}}$, de maneira que facilitasse o estudo estatístico, o qual foi efetuado através da utilização do teste qui-quadrado e análise estatística descritiva.

\section{RESULTADOS}

Tabela 1 - Presença de supranumerários em relação ao sexo nos pacientes da Disciplina de Cirurgia e Traumatologia Buco-maxilo-facial, Faculdade de Odontologia, UFRGS. Porto Ale gre, agosto, 2001.

\begin{tabular}{|l|c|c|c|c|}
\hline \multirow{2}{*}{$\begin{array}{l}\text { PRESENÇA DE } \\
\text { SUPRANUMERÁRIOS }\end{array}$} & \multicolumn{2}{|c|}{ SEXO } & \multicolumn{2}{c|}{ TOTAL } \\
\cline { 2 - 5 } & MASC. & FEMININO & ABS. & $\%$ \\
\hline PRESENTE & 27 & 27 & 54 & $6,4 \%$ \\
AUSENTE & 296 & 498 & 794 & $93,6 \%$ \\
\hline TOTAL & 323 & 525 & 848 & $100,0 \%$ \\
\hline
\end{tabular}

$\mathrm{c}^{2}=3,469(\mathrm{p}=0,063)$

Pelo teste qui-quadrado verificamos que não existe relação entre sexo e incidência de supranumerários. A Tabela 1 apresenta uma igualdade no número de pacientes em relação ao sexo, com a prevalência exata de 1:1. Ao analisarmos o número de pacientes submetidos ao estudo que procuraram a Disciplina de Cirurgia e Traumatologia BucoMaxilo-Facial, encontramos que 525 dos pacientes são do sexo feminino (62\%) enquanto que 323 do total dos pacientes são do sexo masculino (38\%), estabelecendo uma relação de aproximadamente um homem para cada duas mulheres $(1: 2)$.

Em relação aos dados da presença de supranumerários foi possível verificar a presença destes dentes em $6,4 \%$ dos pacientes, referentes a 54 pacientes igualmente distribuídos entre os sexos.

Em relação ao sexo, podemos constatar uma incidência percentual maior de dentes supranumerários no sexo masculino $(8,4 \%)$, enquanto no sexo feminino encontrou-se $5,2 \%$ de incidência.

Em relação às médias etárias encontradas na população estudada, podemos verificar a média de 29.67 anos, enquanto que os pacientes portadores de dentição supranumerária apresentaram a média de 16 anos de idade.

Analisando-se os 54 pacientes com supranumerários, em relação ao sexo e média etária, apresentaram os homens uma média inferior (13.53 anos) em relação ao grupo de mulheres (18.48 anos).

Os dentes supranumerários em sua ampla maioria encontraram-se retidos $(98,4 \%)$. Em apenas 1,6\% dos casos encontramos a presença de um dente supranumerário eruptado.

O estudo apresentou uma prevalência de dentes conóides (cônicos e unirradiculares com desenvolvimento normal) em relação aos dentes classificados como multirradiculares, que englobam os dentes multicuspídeos e odontomas (sem formato regular e raiz rudimentar). Os conóides apresentaram 90,6\% dos casos, enquanto os multirradiculares tiveram uma incidência de $9,4 \%$.

A análise do Gráfico 1 mostra e comprova a maior ocorrência de dentes supranumerários na região anterior da maxila, referente à região de incisivos centrais e laterais, também denominada de pré-maxila. Esta região anatômica apresentou 57,8\% dos casos.

A área de molares superiores (maxilares) foi a segunda região predominante, com $10,9 \%$ dos casos. As regiões de pré-molares da mandíbula e maxila foram igualmen-

$1{ }^{\circledR}$ Microsoft Corporation. 


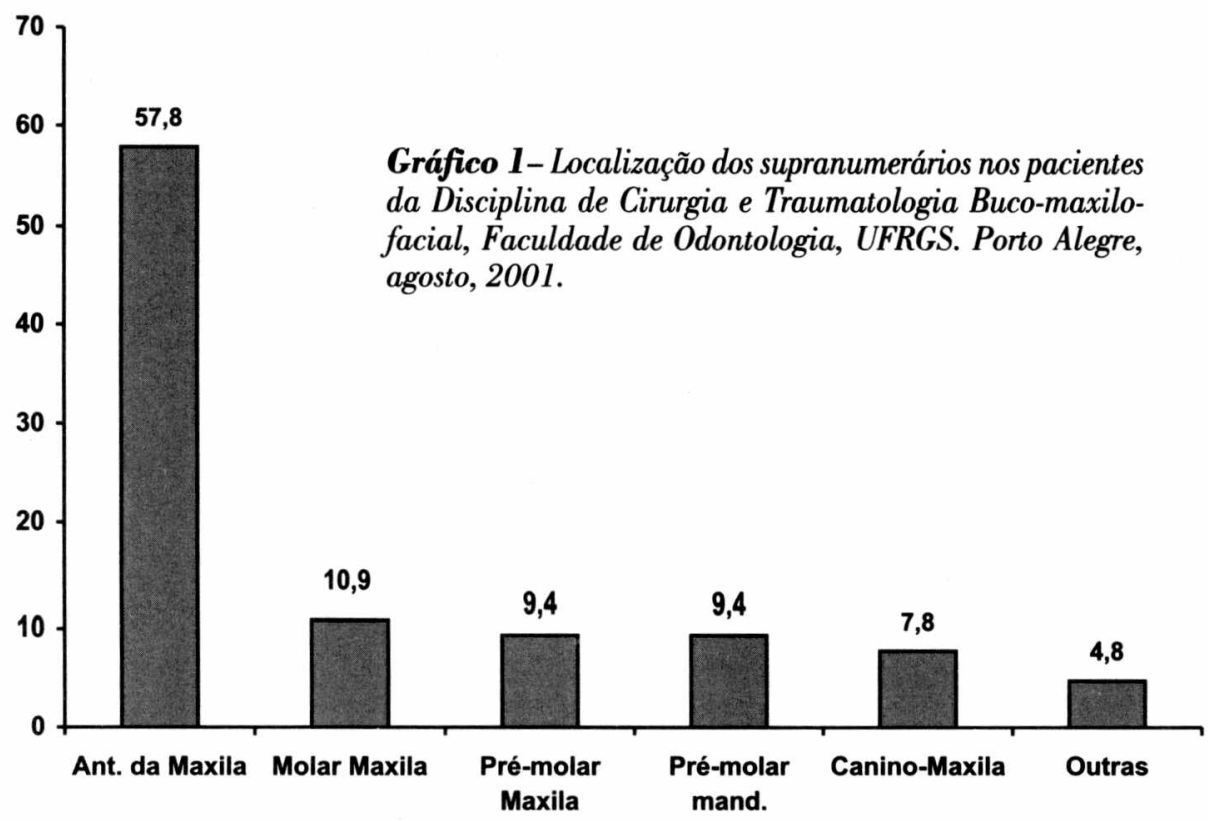

te incididas por $9,4 \%$ dos casos de supranumerários, seguidas pela região de caninos superiores (maxilares) com 7,8\%.

Os demais grupos apresentaram $4,8 \%$ dos casos de dentição supranumerária. Ao considerarmos a distribuição de anteriores e posteriores, considerando a região de canino a canino como anterior e de pré-molares a molares como região posterior, encontraremos a incidência de $68,6 \%$ dos casos na região anterior e $32,4 \%$ na região posterior. Tal diferença determina uma prevalência de 2:1 na relação de dentes anteriores e posteriores.

Ao avaliarmos a distribuição dos casos de supranumerários em relação aos valores totais das duas arcadas por região, encontraremos na região anterior (incisivos) 38 casos (59,3\% do total), seguido pela região de pré-molares com 12 casos $(18,9 \%)$, molares com 8 casos $(12,5 \%)$ e caninos com 6 casos $(9,3 \%)$.

Ao examinarmos a incidência de pacientes com lesão associada aos supranumerários, podemos constatar que $15,8 \%$ dos dentes com supranumerários apresentaram alguma imagem indicativa de aumento do folículo pericoronário e em $84,2 \%$ não foram diagnosticadas imagens compatíveis com patologia. Dos casos com estas imagens a localização foi predominantemente a região anterior da maxila (6 casos) com $9,4 \%$ do total.

Podemos verificar que 10 dentes supranumerários, dos 64 identificados apresentaram imagem sugestiva de alguma patologia. A média etária encontrada nestes pacientes foi de 16,5 anos de idade.

\section{DISCUSSÃ O}

Os dentes supranumerários representam uma importante causa de retenção dentária. A necessidade de um diagnóstico precoce mente inviável, apesar de vermos com restrição esta técnica, devido ao elevado índice de insucesso.

Discordamos de KING et al.(1993) ao afirmarem que o tratamento cirúrgico acarreta algum tipo de seqüela, principalmente aos dentes adjacentes, pois mesmo que algum dano eventual ocorra sobre estes dentes, a manutenção do supranumerário será sempre um fator potencial ao desenvolvimento de alguma patologia maior, como cistos e tumores.

Defendemos uma interação multidisciplinar, com intuito de beneficiar sempre o paciente. Portanto, acreditamos que uma interação da ortodontia com a cirurgia, com o objetivo de remover o dente supranumerário e corrigir eventuais prejuízos no arco dentário, com controles periódicos pós-cirúrgicos, seja a situação desejada, concordando com RIZUTTI (1997).

O progressivo desenvolvimento de denpelos profissionais é fundamental. Portanto, a realização de exames radiográficos panorâmicos da face e outros exames de imagem devem ser solicitados, quando a erupção de um ou mais dentes estiver cronologicamente alterada ou a história médica odontológica indicar.

Discutimos e enfatizamos a necessidade de exames panorâmicos periódicos a partir dos seis anos de idade, concordando com PURICELLI et al. (1991). Segundo PEREIRA, PURICELLI (1981) e PURICELLI et al. (1991), afirmam que o trauma na dentição primária deve ser sempre acompanhado de um rígido controle clínico e radiográfico, pois a rotação do eixo eruptivo do germe do dente permanente deve ser considerada.

$\mathrm{O}$ controle clínico-radiográfico visa impedir ou propiciar a detecção de dentes supranumerários com desenvolvimento tardio, concordando com CHADWICK e KILPATRICK (1993).

Entretanto, discordamos de KING et al. (1993) e ZHU et al (1996) que sugerem orientação aos pacientes portadores de dentes supranumerários, pois não somos da opinião de controles clínico-radiográficos nos pacientes que apresentam condições sistêmicas que viabilizem a intervenção cirúrgica para remoção, já que o controle periódico por parte dos profissionais pode ser prejudicado. Todavia concordamos parcialmente com YAMAOKA et al. (1995-a), quando eles afirmam que os dentes devem ser extraídos entre os 8 e 10 anos de idade, enquanto acreditamos que o procedimento deve ser o mais precoce possível, desde que não acarrete danos aos germes dentários. SHATZ et al. (1993) sugere o autotransplante como uma possibilidade de aproveitamento de um dente supranumerário em alguma região com ausência dentária. Acreditamos ser uma idéia não definitivates supranumerários é freqüente em portadores de síndromes, entre elas a disostose cleido-craniana, sendo recomendado revisões radiográficas periódicas. Entretanto, devemos concordar com CHADWICK e KILPATRICK (1993) que afirmam que a radiografia é um momento, sendo aconselhável avaliar a história de dentes supranumerários prévios no paciente ou na história familiar, assim com uma boa avaliação clínica, principalmente devido ao fato de pacientes portadores de um dente supranumerário apresentarem uma chance $30 \%$ maior de desenvolvimento tardio de mais de um supranumerário. Nos casos onde o paciente opta na não remoção, este deve ser alertado das possíveis seqüelas.

Por razões estatísticas, não consideramos em nosso estudo dois pacientes portadores da síndrome de disostose cleido-craniana, sendo um paciente com nove e outro com quatro dentes supranumerários. Contudo, nossos resultados frente a estes dois pacientes não concordam que portadores desta síndrome apresentam uma maior incidência de dentes supranumerários na região anterior da maxila e da mandíbula, já que dos 13 dentes retidos destes pacientes com síndrome (DCC) ${ }^{3}$, sete dentes estavam localizados na região de pré-molares e molares e apenas seis encontravam-se na região anterior dos maxilares.

Nossos resultados concordam com ZHU et al (1996) e TIMOCIN et al (1994) quanto à situação de retenção dos dentes localizados na região anterior da maxila, ao afirmarem presença de respectivamente $75 \%$ e $80 \%$ de retenção dos dentes localizados nesta região. Nossa análise encontrou $85,9 \%$ dos dentes localizados na maxila, e 14,1\% loca- 
lizados na mandíbula, sendo $98,4 \%$ dos dentes supranumerários com retenção.

A literatura apresenta diversos percentuais de incidência por região anatômica. Apesar dos valores se diferenciarem um pouco, a grande maioria é concordante que a região anterior da maxila é mais atingida por dentes supranumerários. Os resultados apresentados por CHADWICK et al. (1993), TROTMAN et al, TIMOCIN et al (1994), são semelhantes ao deste estudo. Já os resultados de KAYALIBAY et al (1996) e NOIKURA et al (1996) são ainda mais precisos, pois estes autores relatam que 45 a $67 \%$ dos supranumerários são na região anterior da maxila. Estes dados apresentados convergem com os achados por este estudo, que foram de $57,8 \%$ dos supranumerários encontrados na maxila.

Entretanto, algumas variações podem surgir quanto a segunda área de prevalência de dentes supranumerários. Concordamos com KING et al. (1993), que afirmam que a região de pré-molares é a segunda mais comum de encontrarmos estes dentes, pois segundo este estudo a região de pré-molares apresentou $18,9 \%$, seguida da região de molares com $12,5 \%$ e caninos com $9,3 \%$ aproximadamente do total dos casos.

Se analisarmos a região de cada arcada individualmente encontraremos uma maior incidência na região de molares superiores com 10,9\%, concordante com TIMOCIN et al. (1994). Os pré-molares apresentaram-se com 9,4\% em cada arcada, igualmente aos dados de HEDGE et al (1996) e ZHU et al. (1996) que referem de 8 a $10 \%$ na região de pré-molares por arcada. Concordamos também com os índices de menor freqüência, como os $2 \%$ de supranumerários encontrados na região de molares inferiores, já que os encontramos em $1,6 \%$ dos casos.

As afirmativas de TIMOCIN et al. (1994) estão de acordo com o nosso estudo, ao afirmarem que dentes supranumerários encontrados em ambas arcadas são mais frequientes na região de pré-molares seguido da região molar.

Concordamos com GELLIN (1984) ao afirmar que a presença de supranumerários pode ser fator de reabsorção radicular alcançando reabsorções maiores envolvendo dentina e o órgão pulpar, assim como pode provocar a rizólise na dentição decídua como na dentição permanente. Portanto, a indicação de exames de imagem por parte do cirurgião-dentista clínico geral ou odontopediatra deve ser considerada.

Ao analisarmos os resultados de nossa população constatamos uma maior ocorrência de dentes supranumerários na dentição permanente. A média etária geral da população que procurou a Disciplina de Cirurgia e Traumatologia Buco-Maxilo-Facial, duran- te o período de 1998 a 2001 , foi de 29,67 anos, enquanto que a população portadora de dentes supranumerários apresentou uma média etária de 16 anos de idade, portanto com dentição permanente, dados concordantes com NYK-HUSSEIN et al. (1996), em seu estudo sobre as correlações de anomalias da dentição decídua com a dentição permanente, onde apresentam uma incidência de apenas 0,1 a $1 \%$ de dentes supranumerários na população que se encontra na dentição primária. Julgamos procedentes as afirmativas destes autores, as quais alterações na dentição decídua acarretam e apresentam associação com alterações na dentição permanente.

Os resultados estatísticos encontrados por nós são concordantes com CHADWICK et al. (1993), que encontraram dentes supranumerários em 1 a $5 \%$ da população geral e com HOU et al. (1995) que referem a existência entre 0,3 e $6,6 \%$ de supranumerários na população em geral, já que encontramos a presença de supranumerários em 7\% da população estudada.

Concordamos com KING et al. (1993) que afirmam que a maioria dos supranumerários são encontrados na forma única, já que em nosso estudo observamos 33 dentes localizados nas arcadas individualmente $(51,5 \%)$ enquanto na forma múltipla os achados foram de 48,5\%.

Ao analisarmos os dados apresentados por ZHU et al. (1996), e confrontarmos com os resultados deste trabalho, confirmamos a presença de dentes supranumerários aos pares como a segunda maior incidência, seguido de três ou mais dentes. Os resultados de ZHU et al. (1996) foram de 12 e $23 \%$ distribuídos respectivamente na dentição decídua e permanente. Este estudo encontrou 37,5\% dos dentes dispostos aos pares, e 11\% em três ou mais (múltiplos).

\section{CONCLUSÕES}

Após a realização do presente estudo podemos concluir que:

os resultados da população estudada concordam com os achados científicos da literatura;

a região anterior da maxila é o local de maior incidência de dentes supranumerários com $68,74 \%$ dos casos;

$98,43 \%$ dos casos de dentes supranumerários apresentam-se retidos;

dentes conóides apresentaram-se em $90,62 \%$ dos casos;

foi encontrado em $15,62 \%$ dos casos espessamento do folículo pericoronário;

\section{ABSTRACT}

This study reports a radiographic review of 848 panoramic radiographs from the archives of Department of Surgery and
Orthopedics, Faculty of Dentistry of the Federal University, with the purpose of evaluating the presence of supranumerary teeth and their characteristics such as gender prevalence, location, morphology, situation in dental arch, number of roots, age of the patients and occurrence of associated pathoses.

The purpose of this study was to analyse the prevalence of supranumerary teeth in our population, comparatively to the literature. Sixty four (64) supranumerary teeth were founded in 54 patients, with the anterior region of the maxilla having highest occurrence showing $37(57,8 \%)$ of the total of the cases studied with supranumerary teeth; prevalence of conical and impacted teeth; without local associated pathoses; The results found in the present study are in accordance to what is shown in the literature.

\section{KEYWORDS}

Supranumerary; Panoramic Radiographs; Teeth Impacted

\section{REFERÊNCIAS BIBLIOGRÁFICAS}

ANIL, S. et al. Mandibulofacial disostosis: case report. Aust. Dent. J., Sydney, v. 40, no.1, p.39-42, 1995.

ATASU, M. et al. Multiple supernumerary teeth in association with cledocraneal dysplasia. J. Clin. Pediatr. Dent., Birmingham, v. 21, no. 1, p.85-91, 1996.

ATASU, M. et al. Synodontia between maxillary central incisor and a supernumerary incisor teeth: a detal, genetic anddermatoglyphic study. $\boldsymbol{J}$. Clin. Pediatr. Dent., Birmingham, v. 20, no. 3, p.247-51, 1996.

BECKER, A. et al. Cleidocraneal dysplasia: part 2 - treatment protool for the orthodontic and surgical modality. Am. J. Orthod. Dentofac. Orthop., St. Louis, v. 111, p.173-83, 1997.

BERGSTROM, K. An orthopantomographic study of hypodontia, supernumeraries and other anomalies in school children between ages of 8-9 years. Swed. Dent. J., Jonkoping, v.1, p.14557, 1977.

BILGE, O. M. et al. Ectodermal dysplasia: a case with impacted permanent teeth. Aust. Dent. J., Sydney, v. 40, no. 4, p.229-32, 1995.

BUENVIAJE, T.M.; RAPP, R. Dental anormalies in children, a clinical and radiographic survey. J. Dent. Child., Chicago, v.2, p.42-46, 1984. 
CANUT, J. A. Mandibular incisor extraction: indications and long-term evaluation. Eur. J. Orthod., Oxford, v. 18, p.485-9, 1996.

CHADWICK, S. M. et al. Late development of supernumerary teeth: a report of two cases. Int. J. Pediatr. Dent., Oxford, v. 3, p.205-10, 1993.

CHATZ, J. P. et al. Indications of autotransplantation of teeth in orthodontic problem cases. Am. J. Orthod. Dentfac. Orthop., St. Louis, v. 106, no. 4, p.3517, 1994.

CHOW, K.M.; O'DONNELL, D.. Concomitante ocorrence of hipodontia and supernumerary teeth in a patient with Down Syndrome. Spec. Care Dent., Chicago, v. 17, no. 2, 1997.

CRAIG, C. E.. Abnormalities in number and in the eruption path of teeth. Dent. Clin. North. Am., Philadelphia, v.12, p.435-47, 1964.

DIXON, G.H, STEWART, R.E. Genetic aspects of anomalous tooth development. Orofac. Genet., C.V. Mosby Co, St Louis, v.139, 1976.

GIBSON, ACL. Concomitant hypohyperodontia: case report. Br. Dent. J., London, v.123, p.338-9, 1979.

GELLIN, M.E. The distribuition of anomalies of primary anterior teeth and their effect on the permanent successors. Dent. Clin. North Am., Philadelphia, v.28, p.69-80, 1984.

HAAVIKK0, K. Hypodontia of permanent teeth. An orthopantomographic study. Suom Hammaslaeaek Toim, Helsinki, v.67, p.219-25, 1971.

HEDGE, S. V. et al. Late development of supernumerary teeth in the premolar region: a case report. Quintessence Int., Berlin, v. 27, no. 7, p.479-81, 1996.

HOU, G; et al. Ectopic supernumerary teeth as a predisposing cause in localized periodontitis: case report. Aust. Dent. J., Sydney, v. 40, no. 4, p.226-8, 1995.

JASMIN, J. R. et al. Supernumerary teeth in twins. Ora. Surg. Oral Med. Oral Pathol., St. Louis, v. 76, no. 2, p.2589, 1993.

JONES, M. L. et al. Evaluation of occlusion, trauma, and dental anomalies in african-american children of metropolitan head start programs. $J$. Clin. Pediatr. Dent., Birmingham, v. 18, no. 1, p.51-4, 1993.

KAYALIBAY, H. et al. The treatment of a fusion between the maxillary central incisor and supernumerary tooth: report of a case. J. Clin. Pediatr. Dent., Birmingham, v. 20, no. 3, p.237-40, 1996.

KING, N. M. et al. Multiple supernumerary premolars: their occurrence in three patients. Aust. Dent. J., Sydney, v. 38, no. 1, p.11-6, 1993.

KRONMILLER, J. E. Spatial distribution of epidermal growth-factor transcripts and effects of exogenous epidermal growth factor on the pattern of the mouse dental lamina. Arch. Oral Biol., Oxford, v. 40, no. 2, p.137-43, 1995.

LYNHAM, A. Panoramic radiographic survey of hypodontia in Australian defence force recruits. Aust. Dent. J., Sydney, v.35, p.19-22, 1989.

LOH, F.C.; YEO, J.F. Paramolar with bifid crown. Oral Surg. Oral Med. Oral Pathol., St. Louis, v. 76, no. 2, p. 257-8, 1993.

MARAGAKIS, G. M. Crown dilaceration ofpermanet incisors followinf trauma to their primary predecessors. J. Clin. Pediatr. Dent, Birmingham, v. 20, no. 1, p.49-52, 1995.

MELNIK, A. Orthodontic movement of a supplemental maxillary incisor though the mid palatal suture area. Am. J. Orthod. Dentofac. Orthop, St. Louis, v. 104, no. 1, p. 85-90, 1993.

MERCER, A.E. Letter to Editor. Br. Dent. J., London, v.129, p.402, 1970.

NIK-HUSSEIN, N. N. et al. Dental anomalies in the primary dentition: distribution and correlation with the permanent dentition. J. Clin. Pediatr. Dent., Birmingham, v. 21, no. 1, p.159, 1996.

NOIKURA, T. et al. Double dens in dente with a central cusp and multituberculism in bilateral maxillary supernumerary central incisors. Oral Surg. Oral Med. Oral Pathol., St. Louis, v. 82, no. 4, p.466-9, 1996.

PELTOLA, J. S. et al. Radiographic findings in the teeth and jaws of 14- to 17-year-old Estonian schoolchildren in tart and Tallinn. Acta. Odontol. Scand., Oslo, v. 55, p.31-5, 1997.

PEREIRA, C.B.; PURICELLI, E. Recuperação de um incisivo central dilacerado. RGO, Porto Alegre, v.29, n.1, p.5960 , jan./mar., 1981.

PEYRANO, A. et al. Endodontic management of mandibular lateral incisor fused with supernumerary tooth. Endod. Dent. Traumatol., Copenhagen, v. 11, p.196-8, 1995.

PURICELLI, E., CLOSS, L.; BERTHOLD, T.. Incisivo central superior com desvio do eixo de erupção e dilacerado: recuperação ortodôntica-cirúrgica. $\boldsymbol{R G O}$, Porto Alegre, v. 32, n. 2, p. 22-25, novembro, 1991.

RIZZUTI, N. et al. A case of hyperdontia with twenty-two supernumeraries: its surgical-orthodontic treatment. Am. J. Orthod. Dentofac. Orthop., St. Louis, v.111, p.471-80, 1997.

SHAPIRO, S.D.; FARRINGTON, F.H.. A potpourri of syndromes with anomalies of dentition. Birth Defects, New York, v.19, p.129-40, 1983.

SPYROPOULOS, N.D.; PATSAKAS, A.J.. ANGELOPOULOS, A.P. Simultaneou presence of partial anodontia and supernumerary teth. Oral Surg. Oral Med. Oral Pathol., St. Louis, v.48, p.536, 1979.

TASAR, F. et al. Cleidocraneal dysplasia: case report. Aust. Dent. J., Sydney, v. 40, no. 6, p.352-6, 1995.

TIMOCIN, N. et al. Supernumerary molars and paramolars. J. Nihon. Univ. Sch. Dent., Tokio, v. 36, no. 2, 145-50, 1994,

TROTMAN, C. et al. Four maxillary incisors: a case report. Special Care in Dentistry, Chicago, v. 14, no. 3, p.112$5,1994$.

VAN DER LINDEN, W. et al. Diseases and lesions associated with third molars. Oral Surg. Oral Med. Oral Pathol,, St. Louis, v. 79, no. 2, p.142-5, 1995.

VAN DER WEIDE, Y. et al. Symptomalogy of patients with oligodontia. J. Oral Rehabilitation, Oxford, v.21, p.247-61, 1994.

VICHI, M. et al. Eruption anomalies of the maxillary permanent cuspids in children with cleft lip and/or palate. J. Clin. 
Pediatr. Dent., Birmingham, v. 20, no. 2, p.149-53, 1996.

VIERUCCI, S. et al. Dental and craniofacial findings in hypohidrotic ectodermal dysplasia during the primary dentition phase. J. Clin. Pediatr. Dent., Birmingham, v. 18, no. 4, p.291-7, 1994.

WYNNE, S. E. et al. Hereditary gingival fibromatosis associated with hearing loss and supernumerary teeth - a new syndrome. J. Periodontol., Chicago, v. 66, no. 1, p.75-9, 1995.

YAMAOKA, M. et al. Effects of maxillary anterior supernumerary impacted teeth on diastema. Oral Surg. Oral Med. Oral Pathol., St. Louis, p. 252, 1995-a.

YAMAOKA, M. et al. Supernumerary impactions of the mandibular cuspids and bicuspids: case report. Aust. Dent. J., Sydney, v. 40, no. 1, p.34-5, 1995-b.

ZHU, L. et al. Supernumerary and congenitally absent teeth: a literature review. J. Clin. Pediatr. Dent., Birmingham, v. 20, no. 2, p.87-95, 1996.

Endereço para correspondência:

Profa. Edela Puricelli

Rua Quintino Bocaiuva, 465

CEP 90440-051

Porto Alegre-RS, Brasil

Fone: 0 xx 5132226767

e-mail: epuricelli@uol.com.br 